\title{
Different methods for characterizing surface roughness using laser speckle technique
}

\author{
Mayada B. Al-Quzweny ${ }^{1}$, Sabah M. Hadi ${ }^{2}$ \\ ${ }^{1}$ Department of Physics, College of Science, Baghdad University, Baghdad, Iraq \\ ${ }^{2}$ Renewable Energy Directorate, Ministry of Science and Technology, Baghdad, Iraq
}

E-mail: mayada_quzweny@yahoo.com

\begin{abstract}
In this work, results from an optical technique (laser speckle technique) for measuring surface roughness was done by using statistical properties of speckle pattern from the point of view of computer image texture analysis. Four calibration relationships were used to cover wide range of measurement with the same laser speckle technique. The first one is based on intensity contrast of the speckle, the second is based on analysis of speckle binary image, the third is on size of speckle pattern spot, and the latest one is based on characterization of the energy feature of the gray level co-occurrence matrices for the speckle pattern. By these calibration relationships surface roughness of an object surface can be evaluated within these relations ranges from single speckle pattern image which was taken from the surface.
\end{abstract}

Key words roughness, speckle, CCD camera.

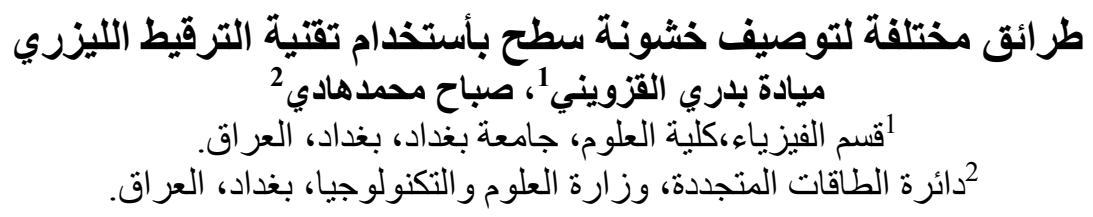

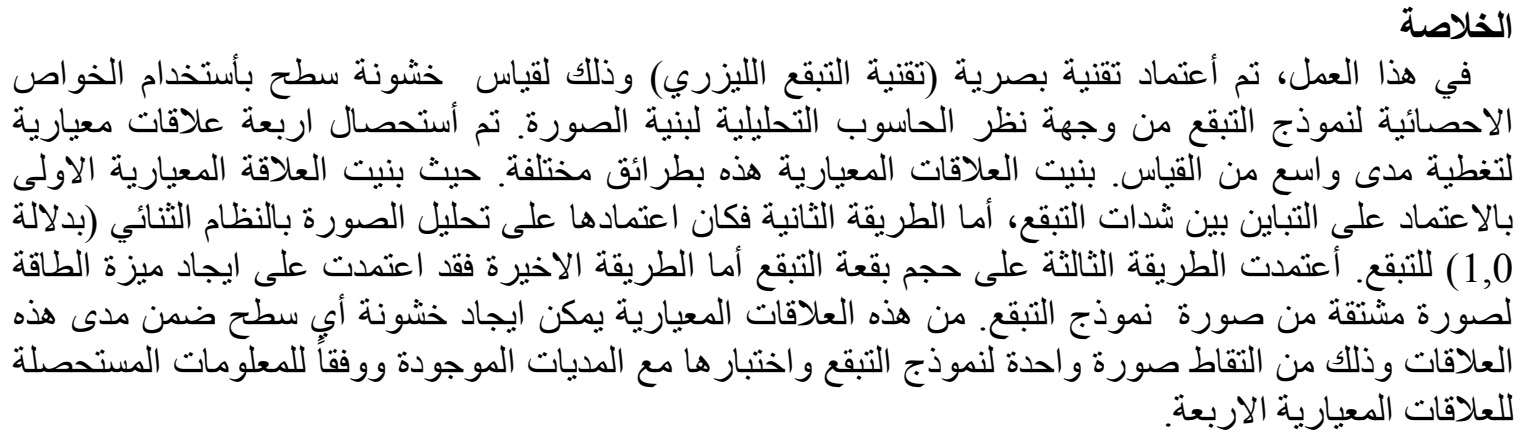

\section{Introduction}

The surface roughness is an important parameter for evaluating the material surfaces since it directly affects on optical and mechanical properties of the materials [1]. The surface roughness measurements based on the stylus instruments have some drawbacks such as possible surface damages, long measuring times and difficulty of in-situ measurement process. These drawbacks have prompted the development of alternative techniques including optical methods such as laser speckle technique 
for the surface roughness measurements as non-contact optical method which leads to non-destructive, fast and continuous measurements[2]. When the laser was invented in the early 1960s the first users noticed, a high contrast grainy pattern when the laser light fell on a matt surface such as paper or unpolished metal or glass. This effect was initially called "granularity" by Rigden and Gordon, but soon after that the name speckle becomes more popular [3-5]. Then from the encyclopedic definition of optical speckle is "a phenomenon in which the scattering of light from a highly coherent source, such as a laser, by a rough surface or inhomogeneous medium generates a random -intensity distribution of light that gives the surface or medium a granular appearance "so, the random-intensity distribution results from the randomly constructive or destructive interference of the scattered light rays[6]. Actually, the speckle pattern contains massive information of the surface geometrical and physical properties[7]. Over the decades, since the invention of lasers; researchers have discussed the relationship between surface roughness and speckle pattern statistical properties as a method for offline as well as on-line surface measurements [8]. This offer a powerful and simple tool for studying surface roughness, for measuring displacement, strain, deformation and vibration analysis [9].

In this work we will investigate the statistical properties of speckle pattern from the point of view of computer texture analysis, and study four measurement methods to obtain a surface roughness from a single laser speckle pattern.

\section{Experimental set up}

The experimental setup is in two stages: first one is preparing and measuring of stainless steel specimens. A set of flat specimens of stainless steel had dimensions $60 \times 40 \times 5 \mathrm{~mm}$ polished with different abrasive papers to acquire different surface roughness. The arithmetic mean surface roughness $\mathrm{R}_{\mathrm{a}}$ of these flat metal ranging from 0.07 to $1.58 \mu \mathrm{m}$ which had been measured by using the stylus profilometer (surface roughness tester TR-100). This device is shown in Fig.1.

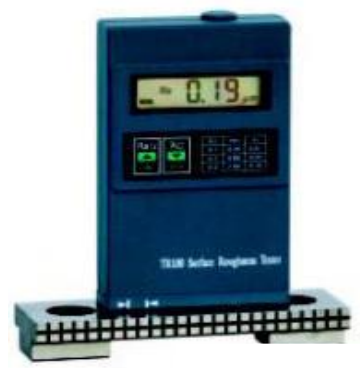

Fig.1: RT(100) stylus profilometer device.

The second stage is building optical system to take speckle pattern image, this optical system configuration of very simple setup, see Fig.2, consisting of $\mathrm{CW}$ diode laser of wavelength of $630 \mathrm{~nm}$. In this experiment the laser which used has some special specifications to match this work, these specifications was high stability output intensity, and of transverse mode TEM $_{00}$ to ensure the laser spot consists of one part and the scattering done by the neighboring elements of the surface. In case of normal incidence, the spot size diameter of the laser beam is 130 pixel depending on the CCD camera that used in this experiment.

Cyber-shot DSC-W610 CCD camera was used, effective pixel numbers of 
camera approximate 14.1 mega pixels this meaning that the resolution is $(4320$ x 3240 ) pixel. The camera was located in normal direction on surface, and the angle between the incident laser beam and the normal direction was fixed. In this setup, the angle was 30 degree, and power of the laser could be adjusted to avoid saturation in digital camera signal.

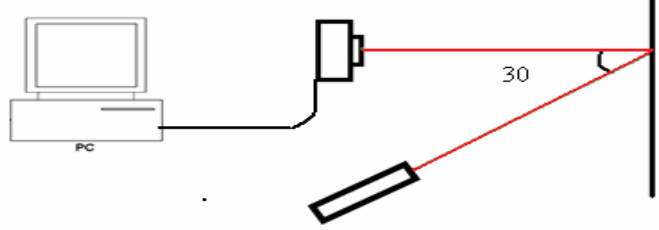

Fig.2: Optical arrangement of the experiment .

By the means of the optical arrangement, different speckle pattern images from standard roughness surface specimens were obtained. Information extraction from texture images can be accomplished by different texture analysis methods, which are classified into four categories: statistical methods , geometrical methods, model -based methods, and signal processing methods $[10,11]$. Those four methods that we used are belong to the statistical methods.

\section{1) Contrast method.}

The speckle pattern images and their surface roughness $R_{a}$ values, which are used to build calibration relationship curve for measure surfaces to $0.25 \mu \mathrm{m}$ are illustrated in the Fig.3.

According to Briers[4], speckle can only be described by statistics and under ideal conditions. Ideal conditions include the same degree of roughness of a surface, allowing the speckle to develop fully, and perfect Gaussian beam statistics, and an air tight environment. The first order statistics of speckle concern deviation from point to point. The assumption can be made that the standard deviation of intensity $(\sigma)$ is equal to the mean of intensity $\langle\mathrm{I}\rangle[6]$.

Where:

$$
<I>=\frac{I_{1}+I_{2}+I_{3}+\ldots \ldots+I_{n}}{n}
$$

and

$$
\sigma=\sqrt{\left\langle I^{2}>-<I\right\rangle^{2}}
$$

In reality, the standard deviation is often less than the mean intensity[12]. The speckle contrast (C) usually defined as the quotient of standard deviation divided by the mean intensity[13,14]:

$\boldsymbol{C}=\frac{\sigma}{\langle\boldsymbol{I}\rangle}$
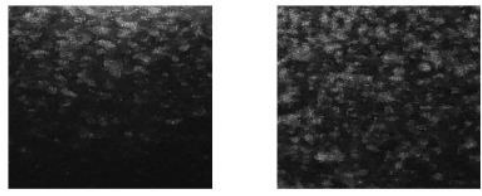

$0.07 \mu \mathrm{m}$

$0.1 \mu \mathrm{m}$
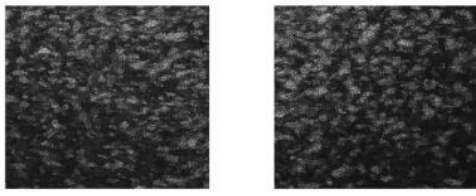

$0.13 \mu \mathrm{m}$

$0.25 \mu \mathrm{m}$

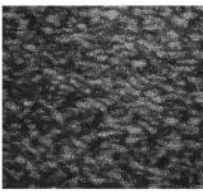

$0.37 \mu \mathrm{m}$

Fig.3: Specklepattern variations against the specimen surface roughness. 
By using equations $(1,2,3)$ digitally five images processing by the MATLAB software in order to find the average, standard deviation and contrast values of speckle intensities of laser speckle patterns could be evaluated as shown in Table 1.

Table 1: Contrast values corresponding surface roughness values at incident angle 30 with non-specular direction.

\begin{tabular}{|l|l|l|l|}
\hline $\mathrm{R}_{\mathrm{a}}(\mu \mathrm{m})$ & $\begin{array}{l}\text { Average } \\
\text { intensity }\end{array}$ & $\begin{array}{l}\text { Standard } \\
\text { deviation }\end{array}$ & contrast \\
\hline 0.07 & 50.0208 & 22.4057 & 0.4479 \\
\hline 0.1 & 76.5739 & 21.7915 & 0.2846 \\
\hline 0.13 & 83.5678 & 22.3296 & 0.2672 \\
\hline 0.25 & 88.1955 & 22.4104 & 0.2541 \\
\hline 0.37 & 91.4614 & 23.7631 & 0.2598 \\
\hline
\end{tabular}

From the Table 1 the calibration curve can be built between contrast of average intensities and standard roughness, see Fig. 4. This calibration curve, within its range, can be used to evaluate surface roughness from single speckle pattern image to $0.25 \mu \mathrm{m}$ because the curve changes its behavior with decreasing at roughness $0.25 \mu \mathrm{m}$.

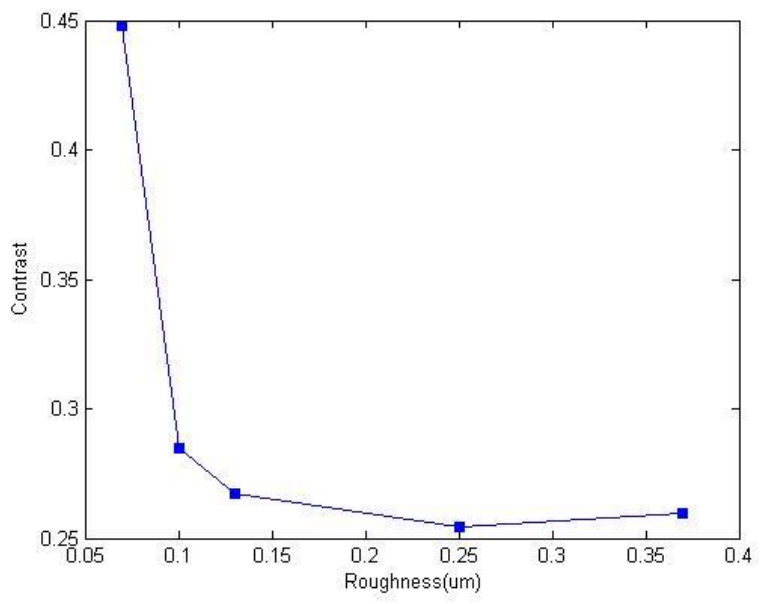

Fig. 4: The contrast of the intensities as a function of the roughness in ( $\mu \mathrm{m})$.

\section{2) Analysis of speckle binary image method.}

To overcome the limitation roughness measurement of contrast method which was used gray image as a data of scattering intensities from a rough surface, another method was used. This method is analysis speckle binary image which used binary image as source of data for scattering intensities. The pixels in binary image with a value of (0) are displayed as black while pixels with the value of (1) are display as white. Parameter of bright regions which was counted and obtained from binary image showed linear relationship with degree of surface roughness. This relationship overcome that limitation from calculating surface roughness in contrast method to $0.94 \mu \mathrm{m}$. The manner to extracted binary images from the CCD camera is illustrated in the Fig.5.

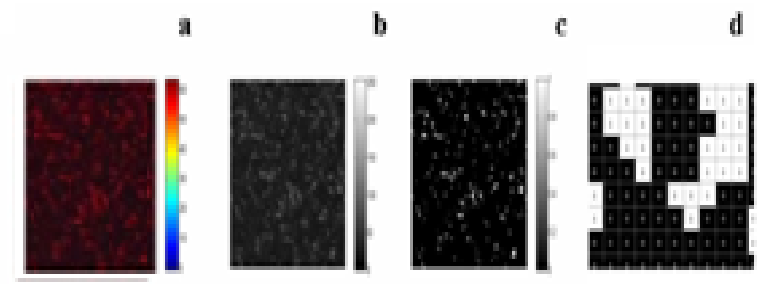

Fig.5 (a) : Apart of speckle pattern in RGB image obtained by using a $C C D$ camera from a surface with $R_{a}$ $0.94 \mu \mathrm{m}$,

(b): Converting the image (a) to gray image,

(c):Convert the image (b) to binary image,

(d): is zoom in the image (c) to show the pixel values.

From experimental setup the CCD camera put with non- specular direction so the intensities of images increased with the increasing of surface roughness, and these increases with intensities affect on the number of bright regions in binary image that extracted by 
image processing. The Table 2 shows these increases of bright region with the increasing of surface roughness.

Table 2: Bright region values corresponding to surface roughness values with non-specular direction ( normal on surface).

\begin{tabular}{|c|c|c|}
\hline Item & $\operatorname{Ra}(\mu \mathrm{m})$ & Bright region count \\
\hline 1 & 0.25 & 3203 \\
\hline 2 & 0.37 & 4467 \\
\hline 3 & 0.63 & 5527 \\
\hline 4 & 0.94 & 7851 \\
\hline
\end{tabular}

The calibration relation between bright regions of standard rough surface is built see Fig.6. The unknown specimens roughness with range 0.25 to $0.94 \mu \mathrm{m}$ can be evaluated roughness depending on this calibration relation, using the same type of material.
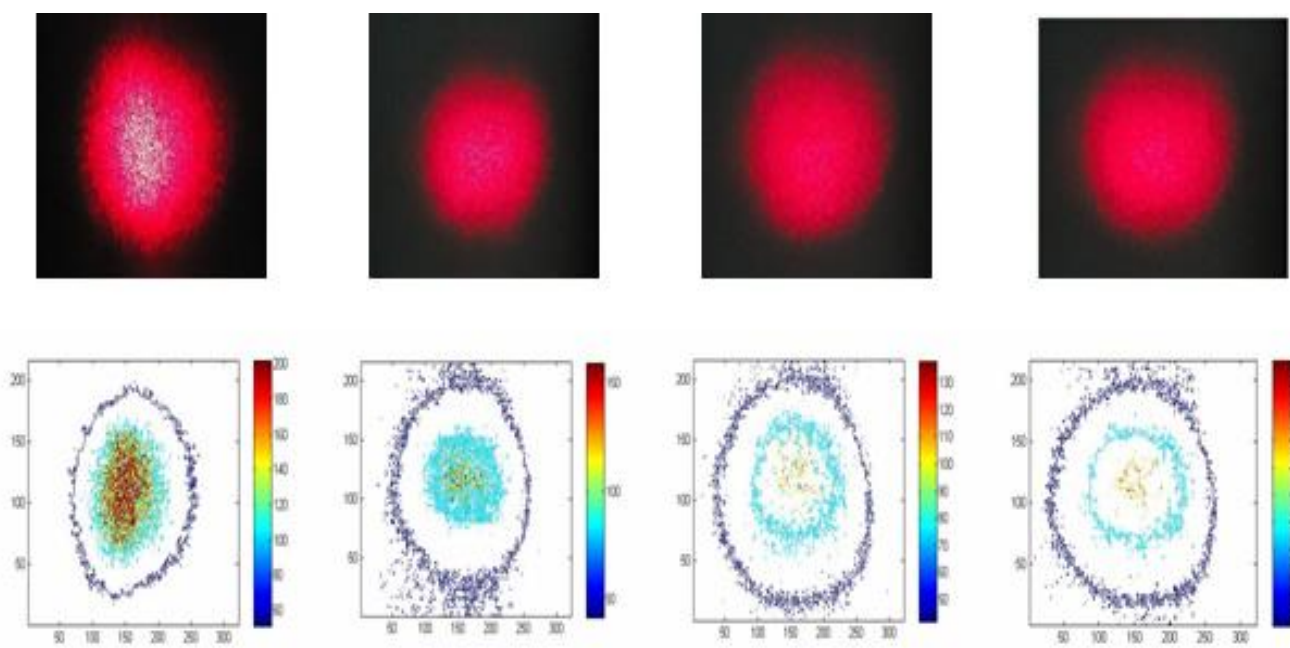

$0.94 \mu \mathrm{m}$

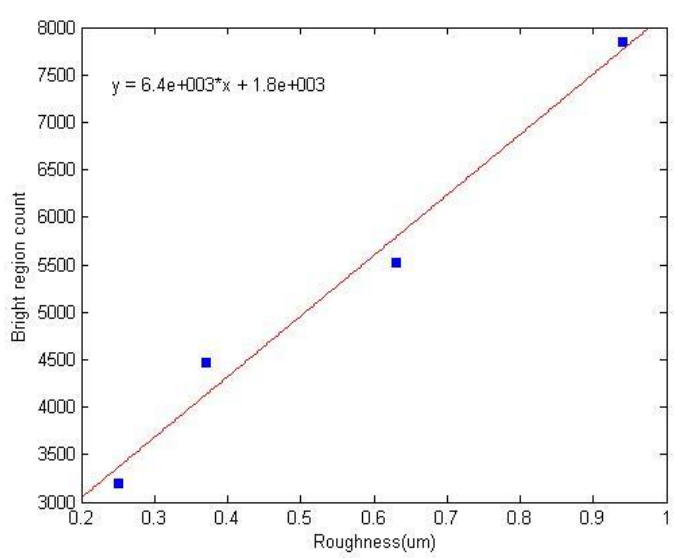

Fig. 6: The number of bright regions counts as a function of roughness.

\section{3) Size of speckle spot method.}

The variation of the spot size with various rough surface was examined to expand the range of measurement. It was found that with increasing roughness of surface the spot speckle size increases. By using MATLAB we could determine the spot size in pixel by using contour and Clable functions as shown in Fig.7.

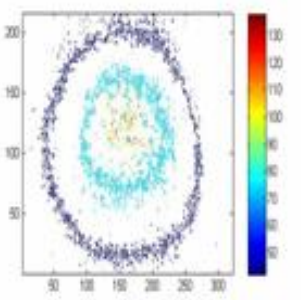

$1.18 \mu \mathrm{m}$

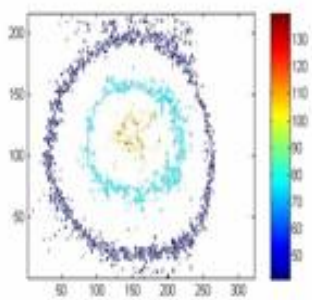

$1.3 \mu \mathrm{m}$

Fig.7: Four images of different spots with its processing. 
laser beam is equal to $30^{\circ}$, the shape of every spot here is an ellipse. This method shows a great potential for non contact optical surface roughness measurements. In our experiment the range with this method reached to 1.3 $\mu \mathrm{m}$. The calibration relationship of this method built between specimens roughness and spot size of speckle pattern as illustrated in Fig.8.

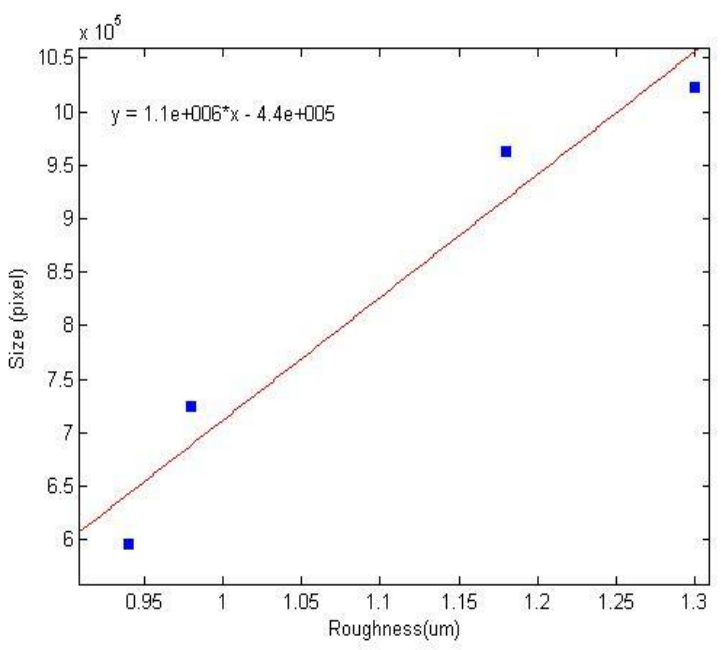

Fig. 8: The counts of pixels as a function of roughness .

\section{4) Energy feature of the gray -level}

\section{co-occurrence matrix method.}

The gray -level co-occurrence matrix (GLCM) deals with the spatial relationships of pairs of gray values of pixels in texture images[7,14]. The Fig.9 shows how the MATLAB function (graycomatrix) calculates several values in the (GLCM) of the 4-by -5 image. Element $(1,1)$ in the (GLCM) contains the value (1) because there is only one instance in the image where two, horizontally adjacent pixels have the values 1and 1. Element $(1,2)$ in the GLCM contains the value (2) because there are two instances in the image where two, horizontally adjacent pixels have the value (1) and (2). Graycomatrix continues this processing to fill in all values in the (GLCM).

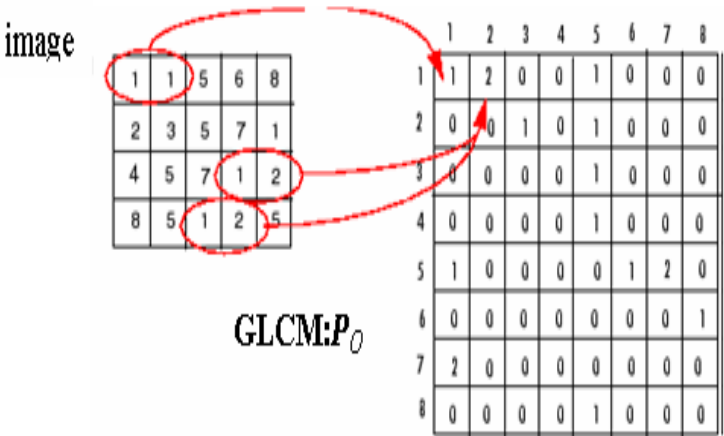

Fig.9: Graycomatrix creates a graylevel co-occurrence matrix (GLCM).

Several GLCM can be computed for several offsets, the way of look at those offsets as an orientation vector $(\boldsymbol{d}, \theta)$, like in polar coordinates, thus $\boldsymbol{d}=1$ and $\theta=\{0,45,90,135\}$ for horizontal, diagonal, vertical, (other) diagonal offset, respectively[3] as illustrated in the Fig. 10.

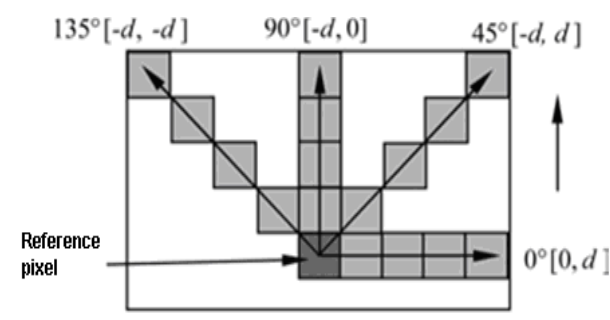

Fig.10: Co- occurrence matrix offset and its direction definition [14].

Haralick et al suggested 14 textural features which can be used [3,14] one of these feature is the energy feature. In many investigation found relationship of the energy feature in speckle pattern with surface roughness reveal that the energy feature may be used to determine 
surface roughness[7]. The energy feature , which is also known as Uniformity or the angular second moment, provides the sum of squared elements in the GLCM . it is defined as follows:

Let $p$ is the GLCM in normalized

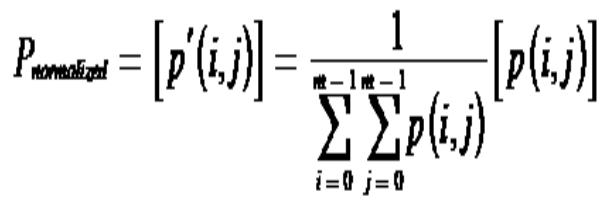

$$
\text { Energy }=\sum_{i=0}^{\mathrm{m}} \sum_{j=0}^{1 \mathrm{~m}} p^{\prime}(i, j)^{2}
$$

Four curves are obtained if drawing the energy feature values against offset $\boldsymbol{d}$ in each direction. Fig.11 show the four energy curves of speckle pattern image of surface with roughness $\mathrm{R}_{\mathrm{a}}=1.3 \mu \mathrm{m}$.

To avoid the influence of differences on evaluating surface roughness, the four energy feature curve values at the same offset are averaged. The average curve of the specimen with $R_{a}=1.3 \mu \mathrm{m}$ shown in the Fig.12. This average curve fitted by the equation:

$$
y=y_{o}+k \exp (-a x)
$$

Where $\mathrm{x}$ indicates the offsets, $\mathrm{y}$ is the energy feature curve values. While $\mathrm{k}$ is an unknown parameter investigated for characterizing surface roughness.
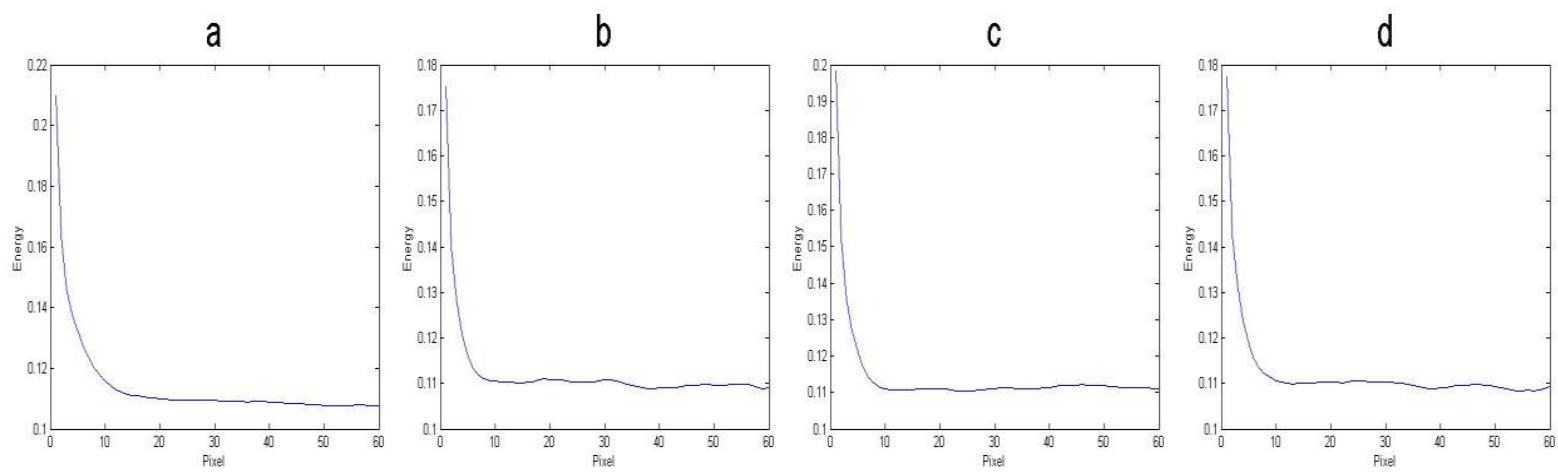

Fig. 11: Co-occurrence energy feature curves of the surface specimen with $R_{a}=1.3 \mu m$.

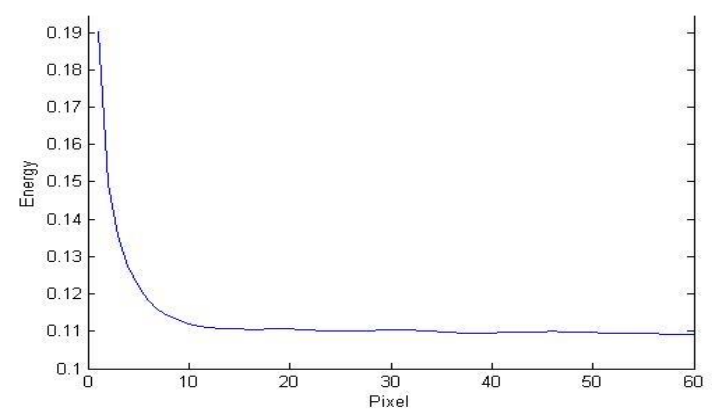

Fig.12: The average curve of the four offset directions of the specimen with $R_{a}=1.3 \mu m$. 
In our experiment, set of other surfaces specimens of different known roughness values were investigated to establish the relationship surface roughness associated and the energy feature curve parameter $\mathrm{k}$ as illustrated in Table 3.

Table 3: K parameter correspond surface roughness values from fitting equations.

\begin{tabular}{|c|c|c|}
\hline item & $\begin{array}{c}\text { Roughness } \\
(\mu \mathrm{m})\end{array}$ & $\begin{array}{c}\mathrm{k} \\
\text { parameter }\end{array}$ \\
\hline 1 & 1.3 & 0.1310 \\
\hline 2 & 1.35 & 0.1362 \\
\hline 3 & 1.42 & 0.1597 \\
\hline 4 & 1.53 & 0.1689 \\
\hline 5 & 1.58 & 0.1706 \\
\hline
\end{tabular}

From Table- 3 the calibration curve can be built between fitting parameter $\mathrm{k}$ and standard roughness, see Fig. 13.

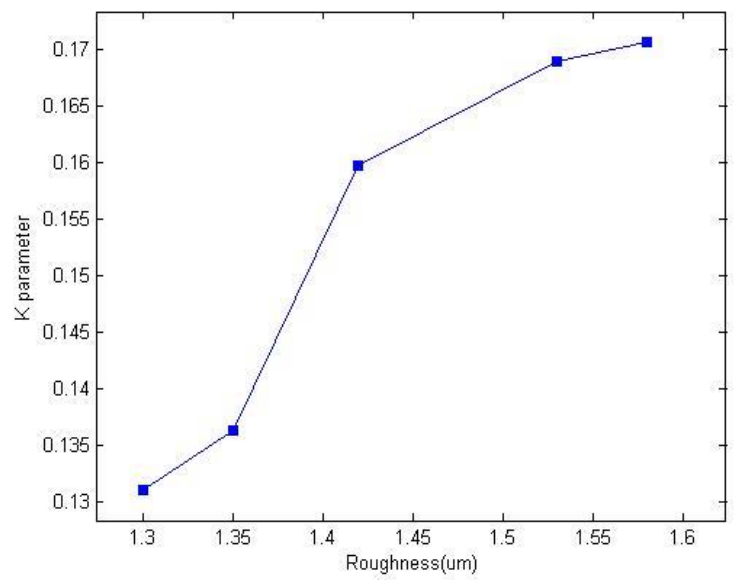

Fig. 13: The relationship between standard roughness values as a function of fitting parameter $(k)$.

Therefore $\mathrm{k}$ is a fitting parameter can be investigated for characterizing surface roughness.

By the aid of these four methods any surface of stainless steel with roughness value between 0.07 to $1.58 \mu \mathrm{m}$ can be evaluated by processing single laser speckle image. The ranges of these four methods under experiment conditions are shown in the Table 4.

Table 4: The four methods and their ranges.

\begin{tabular}{|l|c|}
\hline \multicolumn{1}{|c|}{ Method } & $\begin{array}{c}\text { Range } \\
\text { roughness }(\mu \mathrm{m})\end{array}$ \\
\hline Contrast method. & $0.07-0.25$ \\
\hline $\begin{array}{l}\text { Analysis of speckle binary } \\
\text { image method. }\end{array}$ & $0.25-0.94$ \\
\hline $\begin{array}{l}\text { Size of speckle spot } \\
\text { method. }\end{array}$ & $0.94-1.3$ \\
\hline $\begin{array}{l}\text { Energy feature of the gray } \\
\text {-level co-occurrence } \\
\text { matrix method. }\end{array}$ & $1.3-1.58$ \\
\hline
\end{tabular}

\section{Conclusions}

Different methods used in the present work of laser speckle technique to cover wide range of measurement in laser speckle technique which depend on processing single speckle image. Because every method has a range of measurements. Therefore, to cover a wide range with that technique more than one calibration relationship must be collected and more than one information must be extracted from the single image that was taken from the surface. Each of those information related to one of these methods of speckle technique. The calibrated information must be done with suitable calibration relationship according to surface roughness value where each calibration relationship deal with specific information and with limited range.

\section{References}

[1] M.Chand, A.Mehta, R.Sharma, V.N.Ojha and K.P.Chaudhary, Pure and Appl. Phy. 49 (2011) 335-339. 
[2] E. Kayahan, O.Gundogdu, F. Hacizade, H.Nasibov, IEEE Explore, 978-1-4244- 4210-2 (2009).

[3]H. Fontenelle, Ph.D. Thesis, University of Patras, GREECE ( 2009). [4]J.D. Briers, Optica Applicata. XXXVII (2007) 139-152.

[5] E. Kayahan, H. Oktem, F.Hacizade, H.Nasibov and O.Gundogdu, Trib. Inter. 43 (2010) 307-311.

[6] K.D. Kihm, Advances in heat transfer 30 (1997) 255- 311.

[7] R.S. Lu, SPIE, 6280 (2006) 62801V.

[8] R. S. Lu, G.Tian, D.Gledhill and S.Ward, Appl. Opt. 45 (2006) 88398847.
[9] H.J. Tizinni, Speckle Metrology (Academic Press, Inc., (1978)1st ed.

[10] S. Barra, Ph.D. Thesis, University of New York, USA(2000).

[11] M. Jakobi, Ph.D. Thesis, University of Technical, GERMAN (2000).

[12] J.Ohtsubo and T.Asakura, Optik49, (1977) 445-451.

[13] Z. Tong andX.Chen, Optica Application XLM, 651- 654 (2012).

[14] S. E. Skipetrov, J.Peuser, R.Cerbino, B.Weber, F.Scheffold, Optics Express, 18 (2010) 1419-1435. 